\title{
Estrategias didácticas para el desarrollo del pensamiento crítico en el aula de ciencias
}

\author{
Ángel Blanco López, Enrique España Ramos y Antonio Joaquín Franco-Mariscal \\ Universidad de Málaga. Facultad de Ciencias de la Educación. Didáctica de las Ciencias \\ Experimentales. Málaga (España).
}

[Recibido el 10 de marzo de 2017, aceptado el 24 de mayo de 2017]

Hoy día existe acuerdo sobre la importancia que el pensamiento crítico tiene para los ciudadanos y sobre su consideración como una de las grandes finalidades de la educación científica. No obstante, su desarrollo no está muy presente en la práctica educativa. Este artículo pretende aportar un granito de arena para ayudar a su transferencia a la práctica, delimitando, en primer lugar y como hipótesis de trabajo, un esquema de referencia que pueda ser útil en la enseñanza de las ciencias y que recoge las dimensiones más relevantes planteadas en la literatura. A continuación se presentan tres ejemplos de estrategias didácticas que se han utilizado en la Educación Secundaria y que permiten abordar las dimensiones del pensamiento crítico en el tratamiento de problemas de la vida diaria relacionadas con la salud. Estos ejemplos se centran en el análisis de anuncios publicitarios, en la calidad de las fuentes de información en Internet y en la utilización de los juegos de rol.

Palabras clave: Pensamiento crítico; estrategias didácticas; publicidad; tratamiento de la información; juego de rol.

\section{Teaching strategies for the development of critical thinking in the teaching of science}

Nowadays, there is an agreement on the importance that critical thinking has for citizens and about their consideration as one of the major purposes of scientific education. However, their development still plays a relatively minor role in educational practice. This paper intends to contribute a drop in the ocean to help its transfer to the practice, delimiting, as a working hypothesis, a reference scheme that can be useful for its development in science teaching and including the most relevant dimensions presented in the body of literature. Hereafter, three examples of teaching strategies which have been used in secondary education are presented. These strategies address the dimensions of critical thinking in the treatment of problems of daily life related to health. These examples focus on the analysis of advertisements, the quality of information sources on the Internet and the use of role-plays.

Keywords: Critical thinking; teaching strategies; advertisements; information processing; role play.

Para citar el artículo. Blanco López, A., España Ramos, E. y Franco-Mariscal, A.J (2017). Estrategias didácticas para el desarrollo del pensamiento crítico en el aula de ciencias. Ápice. Revista de Educación Científica 1(1), 107-115. DOI: https://doi.org/10.17979/arec.2017.1.1.2004

Contacto.ablancol@uma.es, enrienri@uma.es, anjoa@uma.es 


\section{Introducción}

Hoy día existe un acuerdo unánime sobre la importancia que el pensamiento crítico (PC) tiene para los ciudadanos (Blanco, España, González y Franco-Mariscal, 2015) y sobre su consideración como una de las grandes finalidades de la educación científica (Osborne, 2014). Su desarrollo está recogido en los currículos actuales en España como uno de los objetivos de la Educación Secundaria (MECD, 2015a) y constituye un aspecto importante de las competencias básicas en ciencia y tecnología, en la medida en que éstas "capacitan a ciudadanos responsables y respetuosos que desarrollan juicios críticos sobre los hechos científicos y tecnológicos" (MECD, 2015b, p.6994).

No obstante, el desarrollo del PC no está muy presente en la práctica educativa debido a varias razones, entre ellas la complejidad del propio concepto (Bailin, 2002) y la dificultad para concretarlo de tal forma que pueda servir de referencia en la práctica docente. A esto hay que unir una extendida visión dogmática de la ciencia, enseñada como un conjunto de hechos que se consideran incuestionables (Osborne, 2014). Los profesores señalan también otras dificultades relacionadas con los contextos escolares (exceso de trabajo, elevada ratio escolar, etc.), mala utilización de las TIC por parte de los estudiantes y falta de formación y recursos (Solbes y Torres, 2013).

Con la finalidad de contribuir a la difusión de recursos que puedan ser de utilidad en las clases de ciencias, este artículo presenta y analiza tres estrategias didácticas que se han llevado a la práctica con estudiantes de Educación Secundaria y Bachillerato en el seno del tratamiento de problemas y/o situaciones de la vida diaria relacionados con la salud. Para ello, en primer lugar se presenta un esquema que puede servir de referencia para el desarrollo del PC.

\section{Un esquema de referencia para el desarrollo del pensamiento crítico}

EI PC está siendo objeto de gran atención en la didáctica de las ciencias y ya se dispone de un buen número de trabajos que plantean cómo entenderlo en la enseñanza de las ciencias o que proponen temas y actividades concretas para su utilización en clase (Vieira, Tenreiro-Vieira y Martins, 2010; Jiménez-Aleixandre, 2010; Oliveras, Márquez y Sanmartí, 2013; Torres y Solbes, 2016). Partiendo de la caracterización que hacen Solbes y Torres (2012) de las competencias que se requieren para el desarrollo del PC en el ámbito de las cuestiones sociocientíficas, se ha identificado un conjunto de ocho dimensiones que se propone de forma tentativa como un esquema de referencia para su desarrollo en la enseñanza de las ciencias desde una perspectiva más general (Tabla 1). 
Tabla 1. Dimensiones del pensamiento crítico (adaptado de Solbes y Torres, 2012)

\begin{tabular}{|l|l|l|}
\hline D1 & \multicolumn{1}{|c|}{ Dimensión (D) } & \multicolumn{1}{c|}{ Descripción } \\
\hline Visión de la ciencia & $\begin{array}{l}\text { Concebir la ciencia como actividad humana con múltiples relaciones } \\
\text { con la tecnología, la sociedad y el ambiente. }\end{array}$ \\
\hline D3 & $\begin{array}{l}\text { Análisis crítico de } \\
\text { la información }\end{array}$ & $\begin{array}{l}\text { Evaluar la credibilidad de las fuentes de información, teniendo en } \\
\text { cuenta los intereses subyacentes. }\end{array}$ \\
\hline D4 & $\begin{array}{l}\text { Tratamiento de los } \\
\text { problemas }\end{array}$ & $\begin{array}{l}\text { Abordar los problemas de forma integral, en su complejidad, } \\
\text { teniendo en cuenta las dimensiones científicas, técnicas, éticas, } \\
\text { culturales, filosóficas, sociales, ambientales, económicas, etc. }\end{array}$ \\
\hline D5 & Argumentación & $\begin{array}{l}\text { Crear argumentaciones sólidas y cuestionar la validez de los } \\
\text { argumentos, rechazando conclusiones no basadas en pruebas, y } \\
\text { detectando falacias argumentativas. }\end{array}$ \\
\hline D6 & $\begin{array}{l}\text { Autonomía } \\
\text { personal }\end{array}$ & $\begin{array}{l}\text { Desarrollar una opinión independiente, adquiriendo la facultad de } \\
\text { reflexionar sobre la sociedad y participar en ella. }\end{array}$ \\
\hline D7 & $\begin{array}{l}\text { Toma de } \\
\text { decisiones }\end{array}$ & $\begin{array}{l}\text { Hacer elecciones racionales y juicios fundamentados como } \\
\text { elementos de las decisiones que emplean para resolver problemas. }\end{array}$ \\
\hline D8 & Comunicación & $\begin{array}{l}\text { Comunicar decisiones usando un lenguaje apropiado, de acuerdo } \\
\text { con el contexto y las metas o intenciones. }\end{array}$ \\
\hline
\end{tabular}

El desarrollo del PC estaría relacionado con el progreso en cada una de las dimensiones anteriores $y$, sobre todo, con la integración de las mismas. Su desarrollo puede fomentarse trabajando individualmente cada una de las ocho dimensiones, pero siendo necesario incluir también problemas en los que se abordan todas ellas de forma integrada.

\section{Ejemplos de estrategias didácticas}

Aunque el PC puede desarrollarse mediante el tratamiento de cualquier tipo de problema (Bailin, 2002), existe bastante acuerdo en la relevancia de los relativos a situaciones de la vida diaria en las que la ciencia y la tecnología juega un papel importante, especialmente sobre los que existen controversias (Torres y Solbes, 2016) y en los que los ciudadanos necesitan tomar decisiones que les afectan personal y socialmente.

En este sentido, se presentan tres estrategias didácticas elaboradas con la finalidad de ayudar a los estudiantes a desarrollar competencias científicas, que se incluyen dentro de un mismo contexto amplio, el de la salud, al que los currículos de secundaria (MECD, 2015a), así como PISA (Franco-Mariscal, Blanco y España, 2017), conceden un papel importante. Para la presentación de cada una de ellas, se comienza analizando su importancia, mostrando a continuación sus características más destacadas y algunas orientaciones sobre cómo utilizarla en las clases de ciencias. Se finaliza analizando su contribución a las dimensiones de PC (tabla 1).

\section{Análisis de anuncios publicitarios}

Los anuncios publicitarios exponen a un buen número de personas en el mundo a una gran cantidad de información científica (Ezquerra y Fernández, 2014) sin que, en muchos casos, sean conscientes de ello. Este hecho, junto con el gran poder de persuasión que tienen, los convierten en un medio potentísimo para influir en las ideas e imágenes de la ciudadanía sobre la ciencia (McSharry y Jones, 2002). Desde la educación científica es necesario abordar explícitamente la ciencia que aparece en la publicidad, con la 
finalidad de que nuestro alumnado esté preparado para comprenderla adecuadamente y desarrolle un PC en el análisis de mensajes informativos y publicitarios (Belova, Chang y Elkins, 2015).

Una de las posibles estrategias consiste en visualizar un anuncio publicitario en clase, en el que la ciencia esté presente (explícita o implícitamente), y llevar a cabo un debate en torno a una serie de preguntas, a partir del que pueden surgir diferentes tareas en función de las respuestas de los estudiantes. En un trabajo anterior sobre el análisis de un anuncio publicitario de un conocido alimento probiótico (Girón, Lupión y Blanco, 2015) se utilizaron las siguientes preguntas:

1. ¿Qué quiere vender? ¿Cuál es el objetivo del publicista?

2. ¿Por qué es bueno para el consumidor lo que quiere vender?

3. ¿En qué se basan para justificarlo?

4. Si queremos indagar para saber si es cierto lo que dice el anuncio, ¿cómo lo haríamos?

Se trata de que los estudiantes sean capaces de identificar la ciencia que está presente en el anuncio y valorar cómo está siendo utilizada. Para ayudar a los estudiantes en estas tareas, además de las orientaciones y explicaciones del profesor se utilizó el Código de Conducta Publicitaria (AUTOCONTROL, 2011). Las preguntas anteriores guardan una relación directa con determinados artículos del código relativos al uso de la ciencia en los espacios publicitarios. Así, por ejemplo, el uso en la publicidad de "Datos técnicos" (art. 17) tiene relación con las preguntas 2 y 3 . Otros aspectos importantes del citado código relacionados con la ciencia son los relativos a las "Garantías" (art. 15), "Ensayos comparativos" (art. 18) y "Testimonios" (art. 19).

Desde la perspectiva del desarrollo del PC, esta actividad incide principalmente en el análisis crítico de la información (dimensión 2; D2), ya que los estudiantes tienen que evaluar la credibilidad de las fuentes de información que se citan en el anuncio, teniendo en cuenta los intereses subyacentes. También se trabaja de forma relevante la argumentación (D5) en la medida en que tienen que cuestionar la validez de los argumentos, valorando si las conclusiones que se presentan en el anuncio están o no basadas en pruebas y detectando, en su caso, falacias argumentativas. La cuestión 4 plantea a los estudiantes la necesidad de estar informado de los temas que se abordan, no limitarse a discursos dominantes y conocer posturas alternativas (D2).

Otras dimensiones del PC pueden también ser abordadas a partir del análisis de anuncios publicitarios. Así, pueden ayudar a los estudiantes a concebir la ciencia como actividad humana con múltiples relaciones, en este caso con el marketing y la publicidad (D1). Solicitar a los estudiantes que tomen una decisión justificada sobre la conveniencia o no de comprar el producto publicitado les proporciona una oportunidad de hacer elecciones racionales y juicios fundamentados como elementos de las decisiones que emplean para resolver problemas (D7) y de comunicar decisiones usando un lenguaje apropiado, de acuerdo con el contexto y las metas o intenciones (D8).

\section{Tratamiento de la información en Internet}

La gestión de la información, el tratamiento que se le da a la misma y la evaluación de sus fuentes se consideran hoy día aspectos importantes de la competencia científica y de la digital. Por ello, es fundamental dotar al alumnado de herramientas y estrategias que le permitan dar una respuesta a una pregunta científica, haciendo uso de 
información proveniente de distintas fuentes. Hoy día Internet se ha convertido en la fuente de información científica que los ciudadanos citan en primer lugar (FECYT, 2014) y el problema ahora, dada la ingente cantidad de información que contiene, es saber identificar la que es de calidad y relevante para su utilización en la resolución de un problema concreto.

Con estos objetivos, en una unidad didáctica sobre salud e higiene bucodental (Blanco, Franco-Mariscal y España, 2016) se planteó al alumnado indagar en la influencia de algunas drogas en la aparición de la caries, teniendo en cuenta la preocupación manifestada por ellos sobre esta cuestión. Los alumnos debían dar respuesta a la pregunta ¿influye el consumo de tabaco, alcohol o drogas en la caries bucal? argumentando a favor o en contra, aplicando los conocimientos científicos estudiados y siendo capaces de discriminar la fiabilidad de la información disponible en la web sobre el tema (Franco-Mariscal, España y Blanco, 2013). El trabajo debía realizarse por parejas, en un plazo de dos semanas, y presentarse como informe escrito y exposición oral en clase. Para llevarlo a cabo, debían elegir una droga (tabaco, alcohol u otra) y consultar, al menos, tres fuentes distintas de Internet, indicando para cada una de ellas los responsables de la web, el grado de fiabilidad que le concedían y la justificación de su elección. Asimismo debían realizar un breve resumen de cada una de las fuentes en un máximo de 10 líneas, indicar las coincidencias y diferencias de las tres fuentes y dar una respuesta razonada y argumentada a la pregunta. Para la exposición oral (figura 1) se podía usar una presentación con un máximo de 10 diapositivas apoyadas en datos e imágenes para explicar sus argumentos. Para dotar al alumnado de algunos criterios con los que evaluar la calidad y fiabilidad de las informaciones de Internet, los estudiantes analizaron con el profesor un artículo científico sobre este tema (Fornás, 2003).

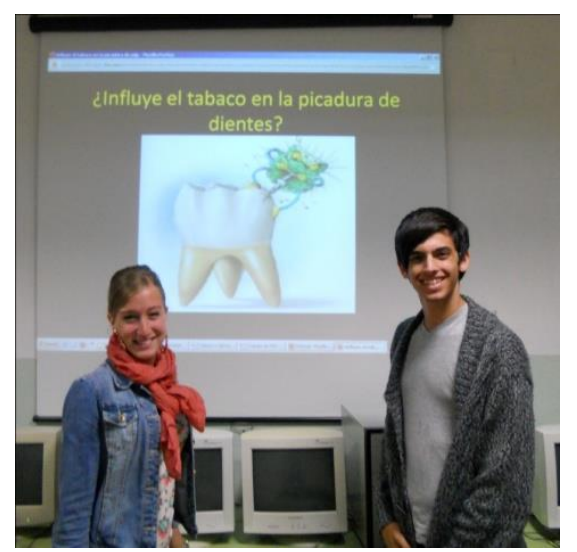

Figura 1. Estudiantes realizando la exposición oral del trabajo realizado sobre la influencia del tabaco en la caries dental

Desde el punto de vista del desarrollo del PC esta actividad incide en seis de las dimensiones planteadas. Los estudiantes trabajan el análisis crítico de la información (D3) al evaluar la credibilidad de las fuentes de información con los criterios facilitados. Por otro lado, abordan el problema de drogas y salud de forma integral (D4) al incluir en su análisis dimensiones de naturaleza científica, ética o social. Por otra parte, las argumentaciones a favor o en contra de la pregunta les llevan a analizar la validez de sus conclusiones y de las pruebas en que se basan (D5). Se pretende asimismo que los alumnos desarrollen una opinión independiente y que, a su vez, sean capaces de reflexionar sobre los hábitos inapropiados de drogas en la sociedad (D6). Del mismo modo, al establecer las conclusiones tienen que ofrecer su elección y toma de decisiones 
ante el problema con juicios argumentados (D7). La exposición pública del trabajo ante sus compañeros permite desarrollar sus capacidades de comunicación (D8), donde también tienen que utilizar un lenguaje científico apropiado y dar a conocer el problema a la sociedad.

\section{Juegos de rol}

Las actividades de juego de rol presentan múltiples ventajas educativas y permiten poner el acento en aspectos procedimentales y actitudinales. A pesar de ello, suelen ser muy poco utilizadas en la enseñanza de las ciencias (McSharry y Jones, 2000). Para desarrollar juegos de rol es necesario partir de un problema inicial que debe ser de actualidad, controvertido, no debe estar resuelto y sobre el que hay información disponible y asequible. Los problemas sociocientíficos se ajustan muy bien a estas características, permitiendo tratar además de cuestiones científicas, aspectos éticos, morales, económicos, sociales, etc. Son problemas que surgen y están relacionados con la ciencia pero que a su vez tienen una compleja relación con la sociedad (Moreno y Jiménez-Liso, 2011).

En un trabajo anterior (España, Prieto y González, 2004) se planteó el juego de rol "juicio a los alimentos transgénicos" como una actividad dentro de una unidad didáctica sobre los alimentos y la alimentación, para estudiantes de 14-15 años. La actividad se desarrolla en tres fases. 1) Fase previa: comprende todos los pasos anteriores a la simulación del juicio, incluyendo la información que facilita el profesor sobre los alimentos transgénicos, las características y reglas del juego y el reparto de cada uno de los roles que se van a representar. A continuación, los alumnos deberán desarrollar el rol que les ha correspondido a partir de las tarjetas que les facilita el profesor, buscando la información correspondiente. 2) Desarrollo del juego: durante una sesión de clase se realiza la escenificación del juicio. 3) Conclusión: para cerrar la actividad, después del veredicto del jurado, el profesor extrae unas conclusiones de lo tratado. Cada alumno elabora un informe basado en la información recibida, la que ha trabajado durante la actividad y las conclusiones personales.

Desde el punto de vista del PC esta actividad proporciona oportunidades para el desarrollo de las ocho dimensiones planteadas. Aborda de forma integral (D4) el problema de la producción y consumo de alimentos transgénicos, ya que en él influyen además de cuestiones científicas, aspectos éticos, morales, económicos, sociales, etc. En este juego también se pone de manifiesto el papel de la ciencia como actividad humana con múltiples relaciones con la biotecnología, relacionadas con la modificación genética y sus repercusiones sociales y ambientales (D1). Un aspecto fundamental de estas actividades es que requieren para su desarrollo el diálogo entre los participantes, llevando así la oralidad al aula (Simonneaux, 2000), tanto durante la preparación del juego como en la escenificación (D8). En la preparación de los roles es fundamental la búsqueda y selección de información, evaluando la credibilidad de las fuentes de información y teniendo en cuenta los intereses relacionados tanto con la postura que se defiende como con la contraria (D3). Los alumnos/as se ven en la situación de identificar los criterios y las posiciones que apoyan tanto su papel como el de los demás, lo que facilita la manifestación de posturas diversas (D2).

Tanto en la preparación de los roles como en las intervenciones durante la escenificación y las conclusiones finales de la defensa y de la acusación, cobra un papel importante la argumentación (D5), relacionando explicaciones y pruebas para crear argumentaciones sólidas a favor o en contra de los alimentos transgénicos y cuestionar 
la validez de los argumentos propios o de los demás, rechazando conclusiones no basadas en pruebas y detectando falacias argumentativas. La realización de actividades de discusión en clase, como el juego de rol, ayuda a los cambios de opinión en el alumnado (Simonneaux, 2000), lo cual lleva a pensar que expresar nuestros puntos de vista u otros contrarios, contribuye a precisar nuestras ideas sobre un problema dado y a desarrollar una opinión independiente, adquiriendo la facultad de reflexionar sobre la sociedad y participar en ella (D6). Se puede resaltar el papel de los juegos de rol al ayudar a identificar los criterios y la información con los que sustentan sus puntos de vista los estudiantes (Simonneaux, 2000). La toma de decisiones (D7) se plantea en diferentes momentos del juego, pero, específicamente, los alumnos que forman parte del jurado, al emitir el veredicto, deben tomar una decisión fundamentada, en este caso sobre la "inocencia o culpabilidad" de los alimentos transgénicos.

\section{Consideraciones finales}

El desarrollo del PC es considerado hoy día una de las grandes finalidades de la educación científica dirigida a la formación de ciudadanos responsables. No obstante, diversos factores influyen en que su práctica no esté muy extendida. Este artículo pretende aportar un granito de arena para ayudar a su transferencia a la práctica. En primer lugar, se ha delimitado, como hipótesis de trabajo, un esquema de referencia que pueda ser útil para su desarrollo en la enseñanza de las ciencias y que recoge las dimensiones más relevantes planteadas en la literatura. En segundo lugar, se han presentado tres ejemplos de estrategias didácticas en el contexto del tratamiento de problemas y situaciones de la vida diaria relacionadas con la salud, analizando su contribución a las diferentes dimensiones consideradas.

\section{Agradecimientos}

Este trabajo forma parte del Proyecto de Investigación de Excelencia "Desarrollo y evaluación de competencias científicas mediante enfoques de enseñanza en contexto y de modelización. Estudios de caso" (EDU2013-41952-P) financiado por el Ministerio de Economía y Competitividad.

\section{Referencias bibliográficas}

AUTOCONTROL (2011): Código de conducta publicitaria. Madrid: Asociación para la Autorregulación de la Comunicación Comercial. Recuperado de www.autocontrol.es/pdfs/Cod_conducta_publicitaria.pdf. Consultado el 13/01/2017.

Belova, N., Chang, S. y Eilks, I. (2015). Advertising and science education: a multiperspective review of the literature. Studies in Science Education, 51(2), 169-200.

Bailin, S. (2002). Critical thinking and science education. Science \& Education, 11(4), 361375.

Blanco, A., España, E., González, F.J. y Franco-Mariscal, A.J. (2015). Key Aspects of Scientific Competence for Citizenship: A Delphi study of the Expert Community in Spain. Journal of Research in Science Teaching, 52(2), 164-198.

Blanco, A., Franco-Mariscal, A.J. y España, E. (2016). A competence-based approach to the design of teaching sequences. An example about Oral and Dental Health and Hygiene. Journal of Biological Education, 50(2), 196-206. 
España, E., Prieto, T. y González, F.J. (2004). Juego de rol sobre los alimentos transgénicos. Un recurso didáctico CTS. En VV.AA (eds.) Perspectivas CiênciaTecnologia-Sociedade na Innovaçao em Ciência (pp. 301-304). Aveiro (Portugal): Universidade de Aveiro.

Ezquerra, A. y Fernández, B. (2014). Análisis del contenido científico de la publicidad en prensa escrita. Revista Eureka sobre Enseñanza y Divulgación de las Ciencias, 11(3), 275-289. Recuperado de: http://hdl.handle.net/10498/16583

FECYT (2014). VII Encuesta de Percepción Social de la Ciencia. Dossier informativo. Recuperado de: www.idi.mineco.gob.es/stfls/MICINN/Prensa/NOTAS.../Dossier_ PSC_2015.pdf. Consultado el 13/01/2017.

Fornás, R. (2003). Criterios para evaluar la calidad y fiabilidad de los contenidos en Internet. Revista Española de Documentación Científica, 26(1), 75-80.

Franco-Mariscal, A.J., España, E. y Blanco, A. (2013).Uso de Internet para analizar las relaciones entre drogas y salud bucodental. Una experiencia en 40 de E.S.O. Capítulo 54 en Soriano, E.; González, A.J. y Cala, V. (eds.), Retos actuales de educación y salud transcultural (1). Almería: Universidad de Almería.

Franco-Mariscal, A.J., Blanco, A. y España, E. (2017). Diseño de actividades para el desarrollo de competencias científicas. Utilización del marco de PISA en un contexto relacionado con la salud. Revista Eureka sobre Enseñanza y Divulgación de las Ciencias, 14(1), 38-53. Recuperado de: http://hdl.handle.net/10498/18845

Girón, J.R., Lupión, T. y Blanco, A. (2015). La publicidad en las clases de ciencias. Análisis de un anuncio sobre un producto probiótico. Alambique, 81, 34-42.

Jiménez-Aleixandre, M. (2010). 10 ideas clave. Competencias en argumentación y uso de pruebas. Barcelona: Graó.

McSharry, G. y Jones, S. (2000). Role-play in science teaching and learning. School Science Review, 82(298), 73-82.

McSharry, G. y Jones, S. (2002). Television programming and advertisements: help or hidrance to effective science education? International Journal of Science Education, 24(5), 487-497.

MECD (2015a). Real Decreto 1105/2014, de 26 de diciembre, por el que se establece el currículo básico de la Educación Secundaria Obligatoria y del Bachillerato. BOE no 3, 3 de enero.

MECD (2015b). Orden ECD/65/2015, de 21 de enero, por la que se describen las relaciones entre las competencias, los contenidos y los criterios de evaluación de la educación primaria, la educación secundaria obligatoria y el bachillerato. BOE no 25,29 de enero.

Moreno, N. y Jiménez-Liso, M.R. (2011). Las controversias socio-científicas: temáticas e importancia para la educación científica. Revista Eureka sobre Enseñanza y Divulgación de las Ciencias, 9(1), 54-70. Recuperado de: http://hdl.handle.net/10498/14624

Oliveras, B., Márquez, C. y Sanmartí, N. (2013). The Use of Newspaper Articles as a Tool to Develop Critical Thinking in Science Classes. International Journal of Science Education, 35(6), 885-905. 
Osborne, J. (2014). Teaching critical thinking. New directions in science education? School Science Review, 352, 53-62.

Simonneaux, L. (2000). Cómo favorecer la argumentación sobre las biotecnologías entre el alumnado. Alambique, 25, 27-44.

Solbes, J. y Torres, N. (2012). Análisis de las competencias de pensamiento crítico desde el aborde de las cuestiones sociocientíficas: un estudio en el ámbito universitario. Didáctica de las Ciencias Experimentales y Sociales, 26, 247-269.

Solbes, J. y Torres, N. (2013). ¿Cuáles son las concepciones de los docentes de ciencias en formación y en ejercicio sobre el pensamiento crítico? Tecné, Episteme y Didaxis, 33, 61-85.

Torres, N. y Solbes, J. (2016). Contribuciones de una intervención didáctica usando cuestiones sociocientíficas para desarrollar el pensamiento crítico. Enseñanza de las Ciencias, 34 (2), 43-65.

Vieira, M.R., Tenreiro-Vieira, C. y Martins, E. (2010). Pensamiento crítico y literacia científica. Alambique, 65, 96-104. 